\title{
Radiation Damage of Tile/Fiber Scintillator Modules for the SDC Calorimeter
}

L. Hu, N. Liu, H. Mao, Y. Tan, G. Wang, C. Zhang, G. Zhang, L. Zhang, Z. Zhang, X. Zhao, L. Zheng, X. Zhong, Y. Zhou and S. Han* IHEP, Beijing, China

\author{
A. Byon, D. Green and A. Para \\ Fermilab, USA \\ K. Johnson \\ Florida State University, USA \\ V. Barnes \\ Purdue University, USA
}

February 1992

*Han took part in the experiment since late March 1991. 


\section{Disclaimer}

This report was prepared as an account of work sponsored by an agency of the United States Government. Neither the United States Government nor any agency thereof, nor any of their employees, makes any warranty, express or implied, or assumes any legal liability or responsibility for the accuracy, completeness, or usefulness of any information, apparatus, product, or process disclosed, or represents that its use would not infringe privately owned rights. Reference herein to any specific commercial product, process, or service by trade name, trademark, manufacturer, or otherwise, does not necessarily constitute or imply its endorsement, recommendation, or favoring by the United States Government or any agency thereof. The views and opinions of authors expressed herein do not necessarily state or reflect those of the United States Government or any agency thereof. 


\title{
RADIATION DAMAGE OF TILE/FIBER SCINTILLATOR MODULES FOR THE SDC CALORIMETER
}

L. Hu, N. Liu, H. Mao, Y. Tan, G. Wang, C. Zhang, G. Zhang, L. Zhang Z. Zhang, X. Zhao, L. Zheng, X. Zhong, Y. Zhou, S. Han* IHEP, Beijing, China

A. Byon, D. Green and A. Para Fermilab, U.S.A.

\author{
$\begin{array}{cl}\text { K. Johnson } & \\ \text { Florida State } & \text { University, U.S.A. }\end{array}$ \\ V. Barnes \\ Purdue University
}

The measurements of radiation damage of tile/fiber scintillator modules to be used for the SDC calorimeter are described. Four tile/fiber scintillator modules were irradiated up to $6 \mathrm{Mrad}$ with the BEPC $1.1 \mathrm{GeV}$ electron beam. We have studied the light output at different depths in the modules and at different integrated doses, the recovery process and the dependence on the ambient atmosphere.

\section{INTRODUCTION}

Detectors operating at the SSC facility will encounter a considerable amount of radiation. It is absolutely critical to establish whether or not plastic scintillators will withstand a radiation dose corresponding to a luminosity of $10^{34} /\left(\mathrm{cm}^{2} \mathrm{sec}\right)$ for a 10 year integrated dose. Without such tests, plastic scintillators cannot be considered as a viable candidate technology for either tracking or calorimetry.

The radiation at the $S S C$ in $p-p$ collisions is due to the neutral and charged pions in "minimum bias" events. Since all the energy of an electromagnetic shower is deposited in a region of limited depth, most of the effects of radiation damage on the calorimeter performance are due to the production of electromagnetic showers in the calorimeter itself, through the $2 \gamma$ decay of $\pi^{\circ}$ 's. These photons will have energies typically in the few $\mathrm{GeV}$ range. This condition allows us to use an electron beam with beam energy of about $1 \mathrm{GeV}$ as an optimum source for the study of radiation damage in the multi-TeV SSC operational environment. We adopted the BEPC (Beijing Electron Positron Collider) electron beam at $1.1 \mathrm{GeV}$ as the radiation source for radiation damage tests. Four tile/fiber scintillator modules were irradiated by up to $6 \mathrm{Mrad}$. We studied their light output at different depths in the modules and at different integrated doses, the recovery process, and their dependence on the ambient environment.

*Han took part in the experiment since late March 1991. 


\title{
2. SCINTILLATING TILE MODULE
}

Four scintillating tile/fiber modules were tested. A standard tile module, as shown in Fig. 1, consists of $21 \mathrm{~Pb}$ plates of absorber, interspaced with 20 scintillator tiles. The scintillating tile is a polystyrene-based scintillator, SCSN81, $2.5 \mathrm{~mm}$ thick. The plate is $5 \mathrm{~mm}$ thick. The area of each tile is about $11 \mathrm{~cm} \times 11 \mathrm{~cm}$ and the $\mathrm{Pb}$ plate is $12.7 \mathrm{~cm} \times 12.7 \mathrm{~cm}$. The light output from the scintillator is collected, using wavelength shifter fibers, BCF91 from Bicron Co., which were directly connected to a photomultiplier tube (PMT) with no depth segmentation.

The tiles were wrapped with aluminum foil and marvel guard paper to optimize the light output and protect the tiles.

The clad optical fibers, containing wavelength shifter, were embedded in the scintillating plastic tiles without glue, in air, at room temperature. Those modules have a $2.5 \mathrm{~cm}$ length of black paint on the fibers, at a $2.5 \mathrm{~cm}$ distance from the PMT. The fibers were glued to a cookie, which was mounted tightly to the PMT with air contact.

All of the $\mathrm{Pb}$ plates, tiles and fibers were enveloped in an aluminum box. The PMT was mounted externally to this box. This arrangement allowed us to control the ambient atmosphere both during irradiation and during annealing.

\section{TEST BEAM AND DOSAGE MONITORING}

The test beam for our tests is the LINAC of BEPC (Fig. 2). The beam characteristics are:

\author{
Beam Particle: electron \\ Beam Energy: $1.1 \mathrm{GeV}$ \\ Beam Peak Intensity: $50-600 \mathrm{~mA}$ (or more) \\ Beam Pulse Duration: $2.5 \mathrm{~ns}$ \\ Pulse Frequency: $12.5 \mathrm{~Hz}$ \\ Beam Spot Size: $1 \mathrm{~cm} * 1 \mathrm{~cm}$ (or $8 \mathrm{~mm}^{*} 5 \mathrm{~mm}$ )
}

Typically, for $600 \mathrm{~mA}$ peak current, $2.5 \mathrm{~ns}$ pulse duration at $12.5 \mathrm{~Hz}$, the beam is about $1.1 \times 10^{11} \mathrm{e} /\left(\mathrm{cm}^{2} \mathrm{sec}\right)$ and is equivalent to an average current of about $18.75 \mathrm{nA}$.

The beam position and spot size can be monitored in the control room by three fluorescent screens, and can be controlled with a set of magnets in the beam line. We also put a fluorescent screen on the front surfaces of modules to monitor the beam position and spot size.

An induced-signal cylinder and BCT (Beam Current Transformer) were put at the end of the beam line. They make the monitoring of both intensity and integrated dose available during irradiation. The beam intensity was roughly monitored by oscilloscope by watching pulses which depend on the intensity and integrated dose through the measurement of the electron flux (in number of electrons). The area of the BCT pulses accurately represents the electron flux. The BCT pulses were sent to an $\mathrm{ADC}$ and gated by the BCT or the induced cylinder pulse. The BCT and ADC both were precalibrated by an accurate pulse generator first, and then calibrated by the 
calibrated by the beam. An IBM/PC computer acquired the ADC data through CAMAC system and converted them into intensity and integrated dosage.

The conversion from incident electron flux to dosage in Mrads at shower maximum is calculated to be, $1.0 \mathrm{rad}=3 \times 10^{6} \mathrm{e} /\left(\mathrm{cm}^{2}\right)$. That means that we define 1.0 Mrad to be $3 \times 10^{12} \mathrm{e} /\left(\mathrm{cm}^{2}\right)$ at $1.1 \mathrm{GeV}$ irradiating the front surface of the modules.

Usually, an irradiating dose rate of $1 \times 10^{11} \mathrm{e} /\left(\mathrm{cm}^{2} \mathrm{sec}\right)$ was used for our test. However, the beam intensity was not very stable. It varied from a maximum of $2 \mathrm{x}$ $10^{11}$, to a minimum of $10^{10} \mathrm{e} /\left(\mathrm{cm}^{2} \mathrm{sec}\right)$.

The integrated beam dose was monitored two ways: by radiochromic film and by alanine. The radiochromic films were incorporated in the test modules, at tile \#4. These films transmit a certain percentage of light according to the dose received. By measuring the transmission before and after irradiation for a certain wavelength with a monochrometer, the approximate dose can be calculated [1]. The alanine method uses 5 pieces of alanine white plastic put on a $10 \mathrm{~cm}^{*} 10 \mathrm{~cm}$ thin Al plate which was inserted on the front face of every test module before irradiating. The radiation effect on the alanine is evaluated by the Electron Spin Resonance (ERS) method at CERN.

We can get very accurate dosimetry by comparing the results from the three methods. We do not regard the dosimetry as the limiting factor in the accuracy of the measurements.

\section{EXPERIMENTAL APPARATUS AND PROCEDURE}

a) Moveable table and module's arrangement

We mounted four tile modules, called tile module \#1, \#2, \#5 and \#6, on a moveable and common support table which was made at FNAL (Fig. 3). The table is motorized and capable of moving in both the horizontal ( $x$ ) and vertical ( $\mathrm{z}$ ) directions (left/right and up/down) by remote control in order to bring the modules into the electron beam and position them precisely. Railway track was laid in the floor of the test beam area to move the table in or out. The speed of the table is about $30 \mathrm{~mm} / \mathrm{sec}$ in the $x$ direction and only about $0.75 \mathrm{~mm} / \mathrm{sec}$ in the $\mathrm{z}$ direction. Modules \#2 and \#5 were at the bottom, and $\# 1$ and \#6 were at the top (Fig. 3).

\section{b) Source Driver and Calibration}

We used a moving radioactive source to calibrate the tile/fiber calorimeter modules. The source is $\mathrm{C}_{\mathrm{S}} 137,6.8 \mathrm{Ci}, 0.38 \mathrm{~mm}$ in diameter and $1.1 \mathrm{~cm}$ in length. It can move at constant speed. The design [2] of the source driver has a remotely actuated driver capable of pushing a fine wire carrying a radioactive source up to 20 feet through any one of a large number of fine tubes into various parts of a calorimeter. Every module has 20 transverse tubes (T1, T2, ..., T20) starting from the side of the modules and 6 longitudinal tubes (L1, L2, ..., L6) starting from the back of the module (Fig. 1). Normally, we only measured the outputs when the source was moving out from the T2, T4, T5, T8, T10 and T18, L2 and L5 tubes. An exception is the T4 tube of module \#5, because the source wire could not pass through the $T 4$ tube. When we observe the annealing process, T1, T2, T4, T6, T8, T10, T14, T18, T20, L2 and L5 were usually used for calibration. 


\section{c) Gas Environment}

Tile modules \#1 and \#2 were kept under nitrogen when irradiated. After irradiating up to $6 \mathrm{Mrad}$, module \#1 was still kept under $\mathrm{N} 2$, but we let air into module \#2 to observe its recovery process in an air atmosphere. Modules \#5 and \#6 were irradiated and annealed in air.

\section{d) Irradiation and Data Acquisition}

The irradiating steps were $0.3 \mathrm{Mrad}, 0.45,0.375,0.375,0.75,0.75,0.75,0.75,0.75$ and $0.75 \mathrm{Mrad}$ up to $6 \mathrm{Mrad}$ in total. When every step was finished, we first bent the beam to the accelerator ring, and then immediately moved the table away from the beam line. After every irradiating step, the modules were measured by the moving source. The PMT current was read out by IBM/PC and CAMAC system via DSP 2032 autoranging scanning DVM when the source was pulled out from the modules. The dark current was measured by picoammeter before and after measurements with the source. When observing annealing, we also used the read out system to get the dark current when the source was in the garage (shielding). The high voltage of the PMTs was on for one half hour at least (usually one hour) prior to the start of data taking. Unfortunately, for this data set we have no absolute calibration system in place.

It is important to emphasize that module \#6 was irradiated to 7.5 Mrad for cosmic calibration before being irradiated along with the other 3 modules. Therefore, module \#6 has been irradiated up to a total of $13.5 \mathrm{Mrad}$.

\section{DATA ANALYSIS AND RESULTS}

We obtained several hundred plots from the data analysis. Here only a few typical plots are shown. As regards data analysis, three points have to be emphasized.

First, we compared the sum of the pedestal plus the dark current from a short run when the source was in the 'garage' (shielded lead box) to the average value of the last 100 event's data from real source run, and found that they were exactly the same. Thus, we usually use the average of the last 50 events data as the sum of the dark current plus pedestal. This sum was subtracted from the data before further data analysis.

Second, we took source data from every transverse and longitudinal tube for every module before irradiating, and defined that to be 0 Mrad data. We found that the outputs are different for different tiles of the same modules, and for different positions ( $x$ ) of same tile. Also, they are not the same for the 20 peaks for a longitudinal scan, corresponding to the 20 tiles of a module. Therefore, we always normalized the data to pre-irradiation data ( 0 Mrad data). This means that we assume that the outputs before irradiation were the same everywhere in a module. We also assume that the PMT was stable.

Third, due to the cycling off and on of the high voltage during irradiation, we made a second normalization to tile \#18 (transverse scan) or peak \#19 (longitudinal scan), because tile \#18 or peak \#19 are assumed to have only little damage. This normalization was used because a second, absolute, normalization was not available. This deficiency will be fixed in subsequent data runs. All data shown here will use 
this relative normalization. Initial indications from subsequent data are that this method is indeed plausible.

\section{a) Transverse Scan of Source}

Since the source speed varies, the width of the output 'broad peak' of the transverse profile varies. We used two ways to define the 'edge' of tiles. One way is to define 'edges' of tiles as the points, there output drops by $50 \%$, as compared to the value in the center. The other way is to define the point where we have the maximum differential value. We found that the results are the same using the two methods. We plot the outputs at various positions ( $x$ ) across tile for different doses. Fig. 4(a) and (b) are two typical plots, for module \#5 tile \#2 and tile \#18.

To get a clearer display of transverse non-uniformity of response caused by the radiation damage, we plot the ratio (normalized to pre-irradiation) of the response at various positions ( $x$ ) across the tile at different doses (Fig. 5 (a) (b) (c) (d), Fig. 6 (a) (b) (c) (d), Fig. 7 (a) (b) (c) (d)). From these plots, we find that tiles at or near shower maximum are quite damaged and yet the transverse uniformity of response is still quite good except for very high doses.

The attenation length for blue light in the tile may decrease due to radiation damage. This would decrease the light yield and potentially cause a non-uniformity of response across the tile. This data indicates that the effect is small. This effect may be due to the long optical path length which effectively integrates over the entire tile.

\section{b) Longitudinal Scan of Source}

First, we have to identify 20 peaks, which correspond to the 20 tiles of a module; see Fig. 8 for a longitudinal scan. The back (called the 20th peak) is the easiest to identify, especially for large dose data, because the large light loss in front means that it is difficult to say where the front peaks are. Although the speed of the source is not always the same, (and thus the spacing of the peaks is not exactly equal) the difference between two peak's spacing is very small.

We first take the highest measured value as a measurement of the peak. We plot the ratio of peak height after some dose, to the peak height before the irradiation for different tiles, i.e., we find the relative output as a function of tile number. This procedure gives us an effective damage profile as a function of the depth in the calorimeter at different doses.

In order to decrease the effect of a lack of absolute normalization, we then normalize these peak's data to tile \#19 peak data for each dose. We did not use peak \#20 for normalization because it is too near the edge. Module \#5 shows much less damage than modules \#1 and \#2. The data is shown in Figure 9. Module \#6 is not discussed further. Module \#5 shows damage (in air) up to $80 \%$ light loss at shower maximum, immediately after irradiation. Module \#1 and \#2 show much worse damage, but they were both irradiated in $\mathrm{N} 2$ and so had no chance to anneal rapidly.

\section{c) Annealing}

Some of the radiation damage is permanent, some can be annealed [3]. After irradiation was stopped, we continued to take data by scanning the source into both the transverse tubes and the longitudinal tubes every day. 
For the longitudinal scan, we plot 0 Mrad and 6 Mrad (after irradiation, annealing 0 day) data with annealing data at $2,3,13$ and 30 days together, for different modules. The data are normalized to peak $\# 19$ and are shown in Figure 10 (Mrad \#2 had 64 days recovery time).

From these plots, we see that the annealing rate depends very much on the gas condition. For modules \#2, \#5, \#6, the recovery process is in air and is largely completed in only 2 days. The outputs achieved a short term plateau in less than 3 days. The plateau value is the same for module \#5 and module \#2 showing that annealing in air leads to the same damage profile for fast irradiation in air or N2. For module \#1, which we always kept in N2 when irradiating or annealing, the annealing process is much slower and still continues 30 days later. Note that after 30 days all 3 modules have essentially the same damage profile. These studies continue as we look for annealing with longer time constants. After 30 days, all modules exposed to 6 Mrad lose about $75 \%$ of their light at shower maximum.

\section{d) Data Fitting}

A method to alleviate the damage using a combination of calibration and correction on an event by event basis using the presumed 2 fold longitudinal segmentation of the EM calorimetry has been developed [4]. We try to fit our results in order to see if the assumptions made in developing that correction technique are valid.

We assume a form; (1-damage $)=\exp (-$ dose $/($ dose $) 0)=\exp (-\mathrm{D} / \mathrm{Do})$. Damage is defined to be the fractional light loss at shower maximum shown in Figure 9 for module \#5. Note that damage refers to the unannealed value for irradiation in air. The fit curves are shown in Figure 11. The fitted value of the characteristic damage dose is $D o=(2.5 \pm 0.5)$ Mrad. The implication for 100 year operation of the barrel calorimeter of SDC, $<0.6 \mathrm{Mrad}$, is damage $<21 \%$ light loss at shower max. This damage does not degrade the resolution of the SDC EM calorimeter [4].

In order to see if the hypothesis [4] of local damage is correct, the damage profile in depth (see Fig. 9) for module \#5 was studied. One needs to know the relation between dose (D) and depth $(t=X / X o)$. We assume that the observed dose at depth $t, D(t)$, is proportional to the energy deposit from the EM shower, $d E(t)$.

$$
\begin{aligned}
d E(t) & =-\left((b t)^{a-1} e^{-b t}\right)(b d t) \\
& \propto D(t)
\end{aligned}
$$

Further, we assume that the light loss is proportional to the exponential of the dose, with some characteristic dose, Do.

$$
\begin{aligned}
\operatorname{Output}(t) & =\operatorname{Exp}[-D(t) / D O] \\
& =\operatorname{Exp}\left[-\left(c(b t)^{a-1} e^{-b t}\right) / D \circ\right]
\end{aligned}
$$

Where $\mathrm{c}$ is a normalizing constant. 
Therefore, the form $f(X)=\exp (-(1 / P 3) *((P 2 * X) * *(P 1-1) * \exp (-P 2 * X))$ was used to fit our depth profile curve, where $a=P 1, b=P 2, C / D o=1 / P 3$.

The data and fit for module \#5 at $1.125 \mathrm{Mrad}$ (corner of the barrel for 100 years at design luminosity) are shown in Fig. 12. The significance of the fit is good, and the fitted values of the parameters are reasonable.

\section{CONCLUSION}

(a) The scintillating tiles near shower maximum are quite damaged after irradiating up to 6 Mrad. After short term annealing, the output at shower maximum is reduced to about $25 \%$ of the initial output.

(b) Recovery in air is much faster than in $\mathrm{N} 2$.

(c) The presence of air during the irradiation inhibits some non-permanent radiation damage (module \#5). However, after 30 days, no differences were observed for air/N2 during irradiation/annealing.

(d) No significant transverse nonuniformity is introduced by doses up to 6 Mrad.

(e) It is important to try other scintillating materials and PMT. A solution for the end cap ( $<50 \mathrm{Mrad}$ ) is not yet in hand. We also plan to establish calibration or correction functions to alleviate the damage.

\section{REFERENCES}

[1] K. Johnson, Internal Report;

[2] V. Barnes and A. Laasanen, Calibration of Scintillating Calorimeters Using a Moving Radioactive Source, Presented at the International Conference on Calorimetry, 1990;

[3] C. Zorn, Designing a Radiation-Hard Plastic Scintillator for High Luminosity Hadron Collider, Proceedings of Workshop, 1990, at Florida State University;

[4] D. Green and A. Para, Radiation Damage, Calibration and Depth Segmentation in Calorimeters, FN 565, 1991. 


\section{FIGURE CAPTIONS}

Fig. 1 Scintillating tile/fiber module

Fig. 2 Test Beam of BEPC

Fig. 3 Moveable table and test module arrangement

Fig. 4 Outputs at various positions ( $x$ ) across the tile

Fig. 5 Relative output (normalized to before-irradiation) at various positions (x) across tile \#2 for modules \#1, 2, 5 and 6 , and for different doses.

Fig. 6 Relative output (normalized to before-irradiation) at various positions (x) across tile \#6 for modules \#1, 2, 5 and 6, and for different doses.

Fig. 7 Relative output (normalized to before-irradiation) at various positions (x) across tile \#18 for modules \#1, 2, 5 and 6, and for different doses.

Fig. 8 Longitudinal source scan of a test module with 20 layers of scintillator.

Fig. 9 Depth profile of damage (normalized to peak \#19) for various different doses.

Fig. 10 Annealing plots from longitudinal scan data (normalized to $0 \mathrm{Mrad}$ and 'peak' \#19 both)

Fig. 11 Fitting curves for 'relative output vs doses'

Fig. 12 Fitting depth profile curve at $1.125 \mathrm{Mrad}$ 

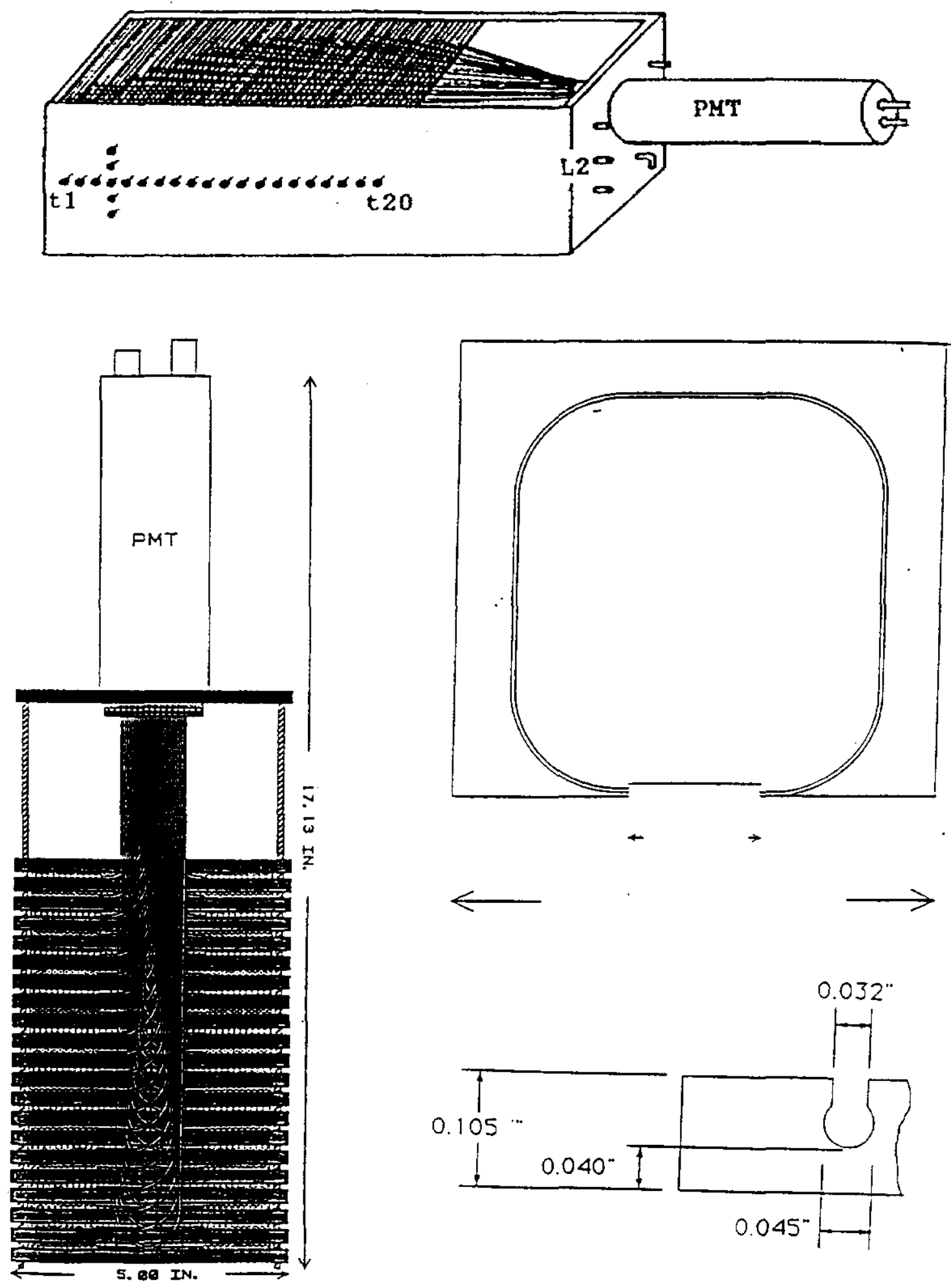

Fig. 1 Scintillating tile module 


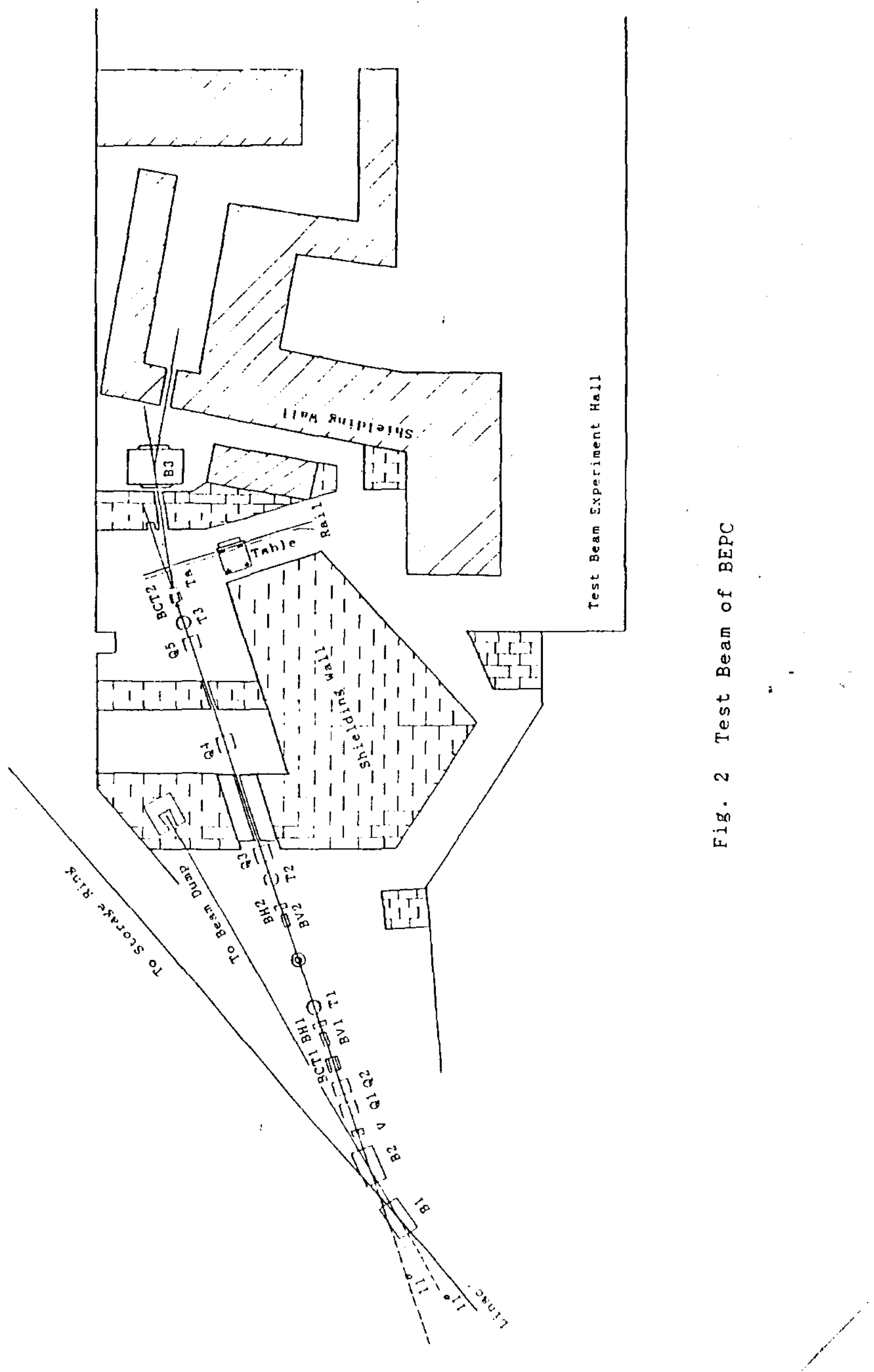




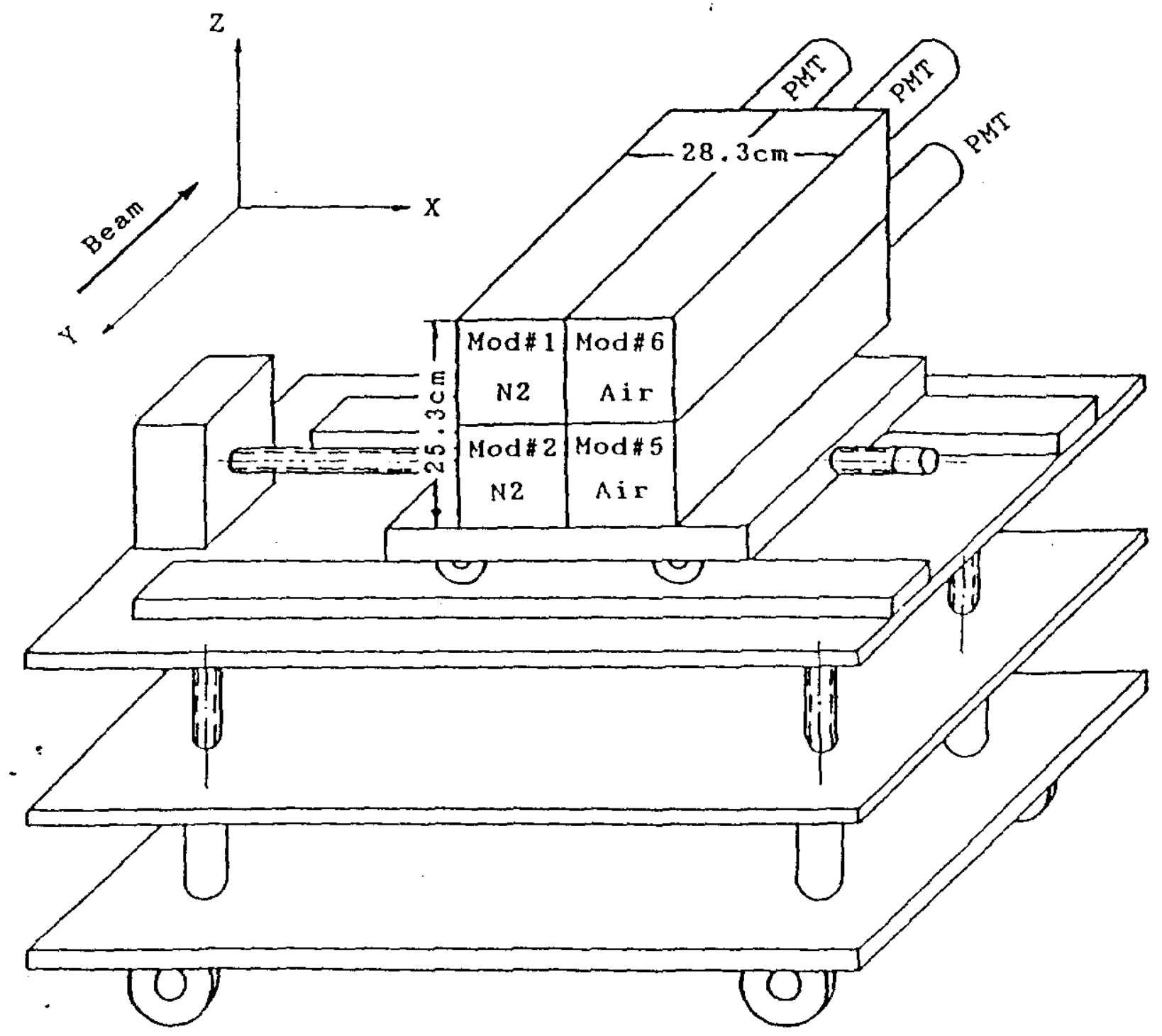

Fig. 3 Moveable table and test module arrangement 
Module \#5 T2 AIR NO BLACK

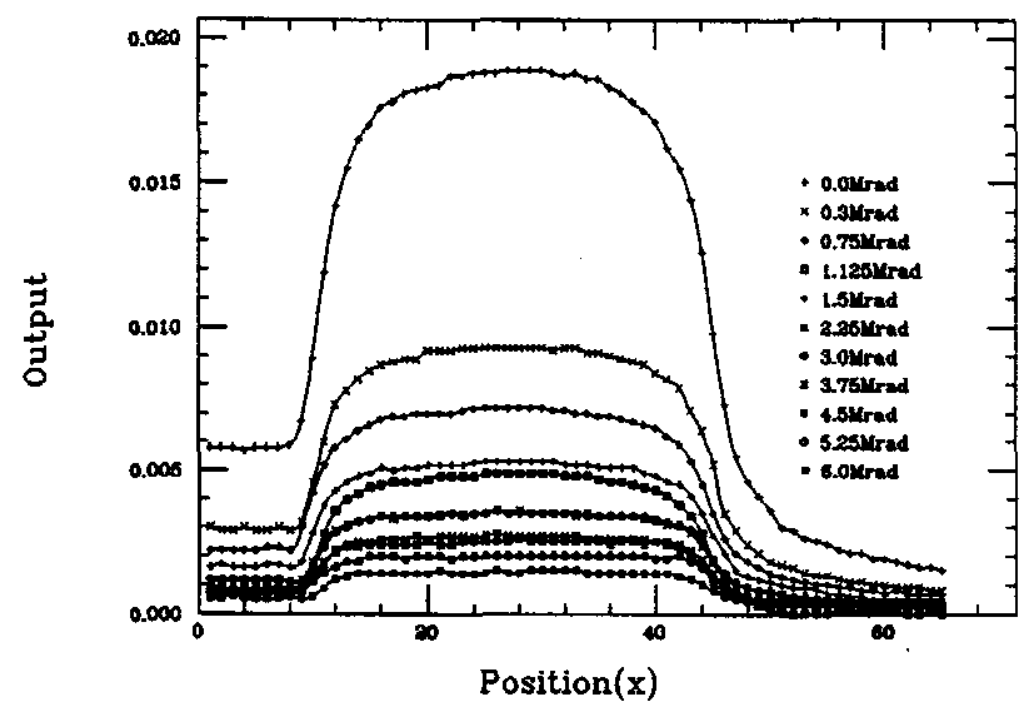

Module \#1 T2 N2

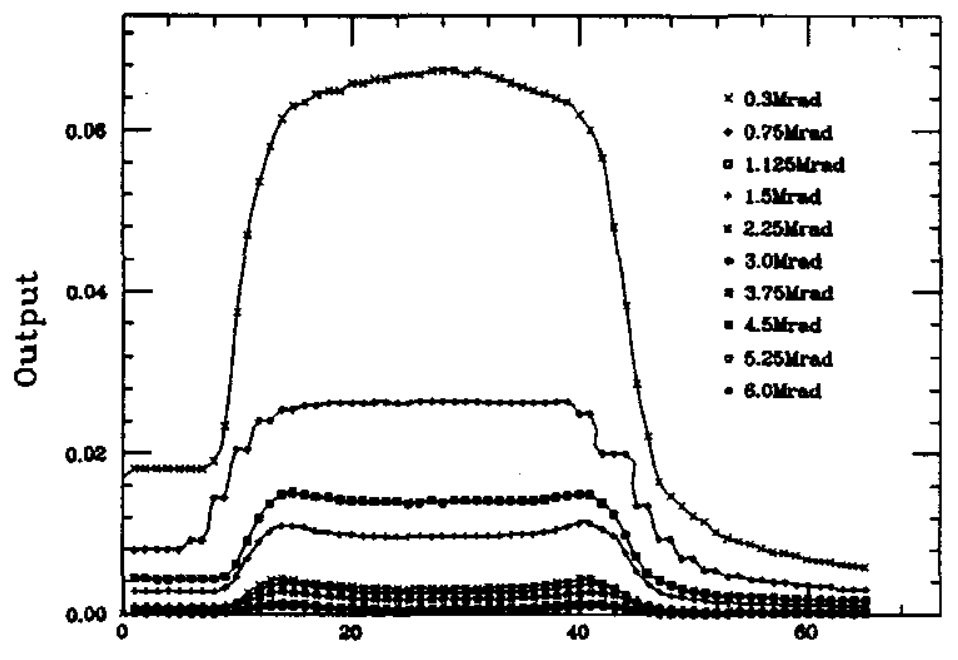

Position( $(x)$
Module \#5 T18 AIR NO BLACK

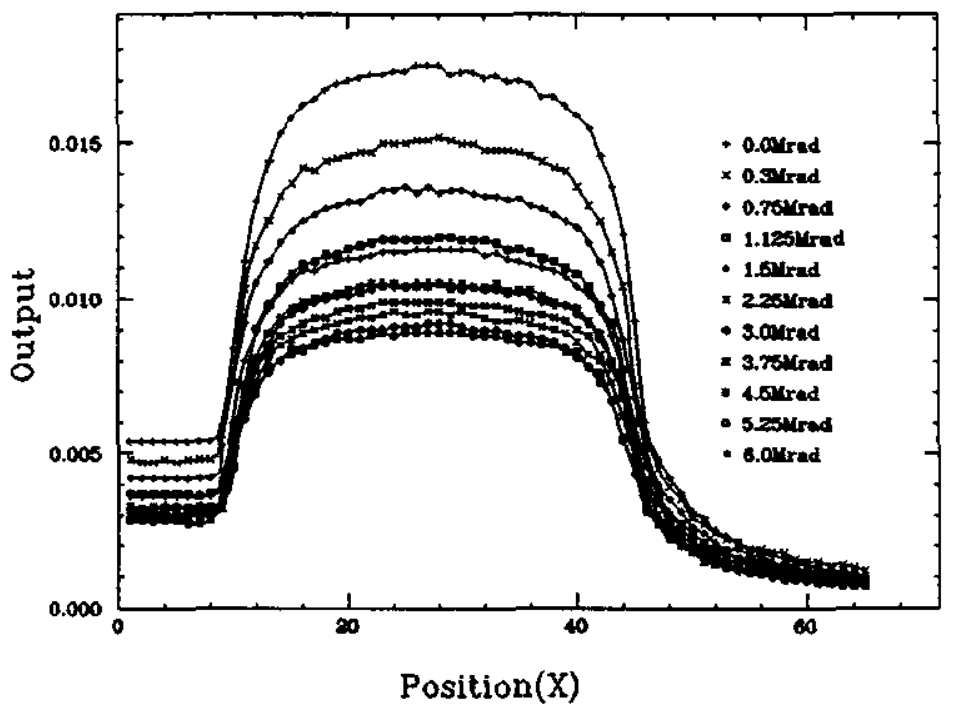

Module \#1 T18 N2

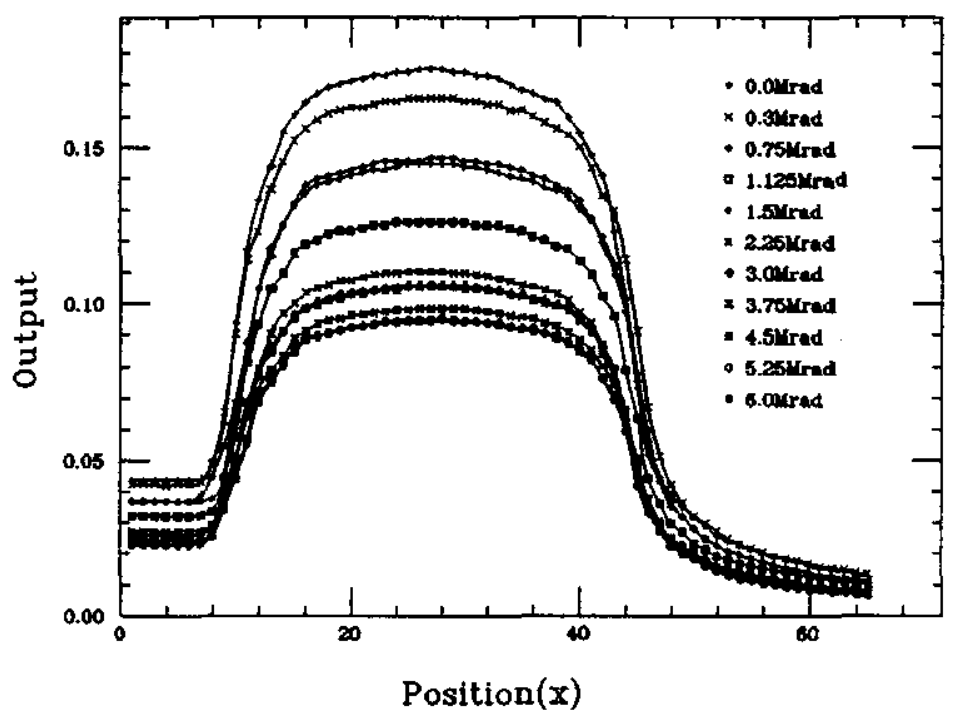

Fig. 4 Outputs at various position ( $x$ ) across the tile 
Module \#1 T2 N2

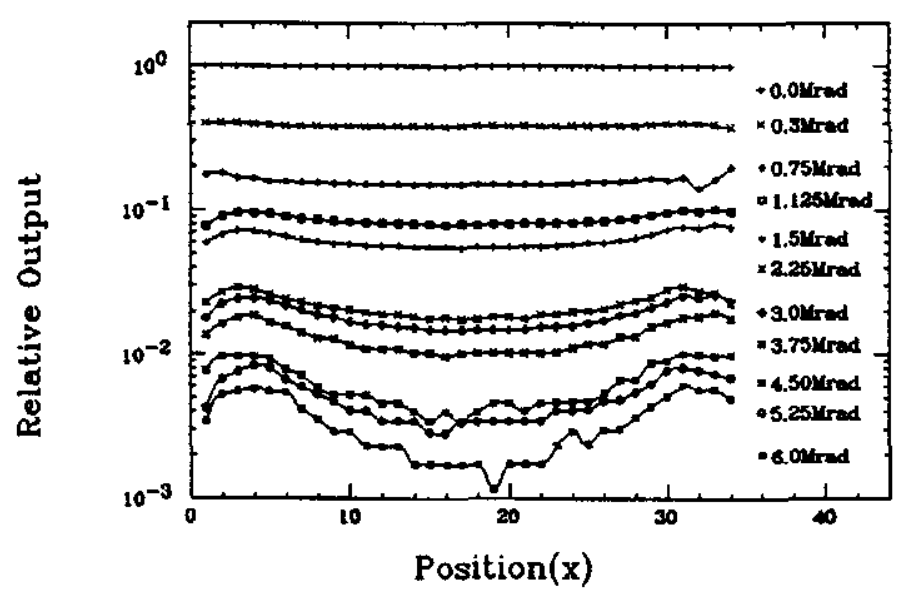

Module \#5 T2 AIR NO BLACK

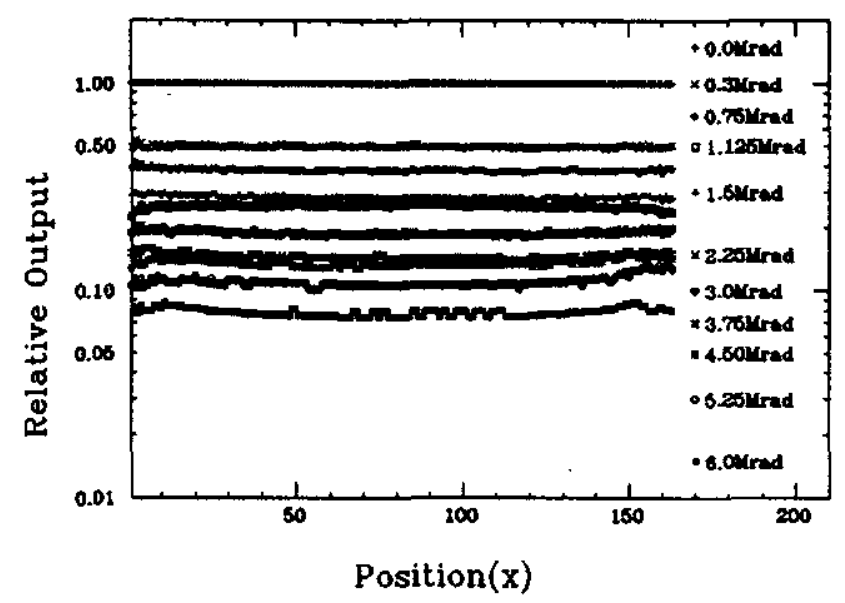

Module \#2 T2 N2
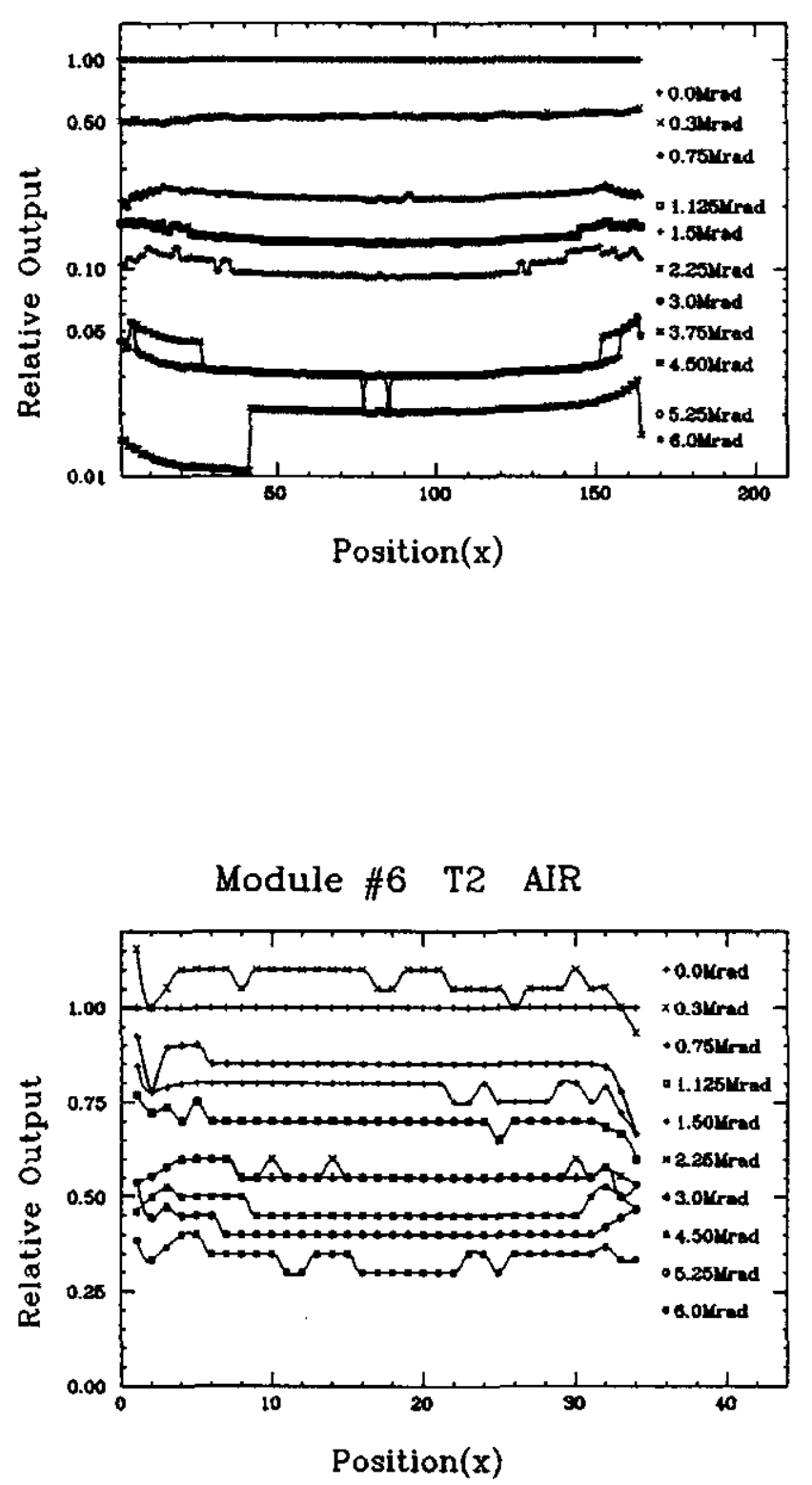

Fig. 5 Relative output (normalized to before-irradiating) at various position ( $\mathrm{x}$ ) across the tile $\# 2$ for module $\# 1,2,5$ and 6 , for different doses. 
Module \#1 T6 N2

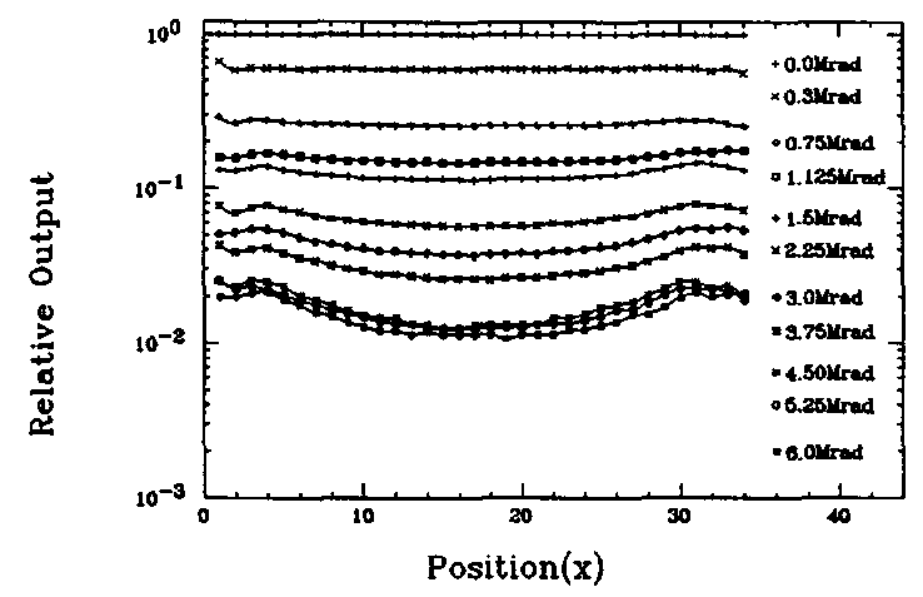

Module \#5 T6 AIR NO BLACK

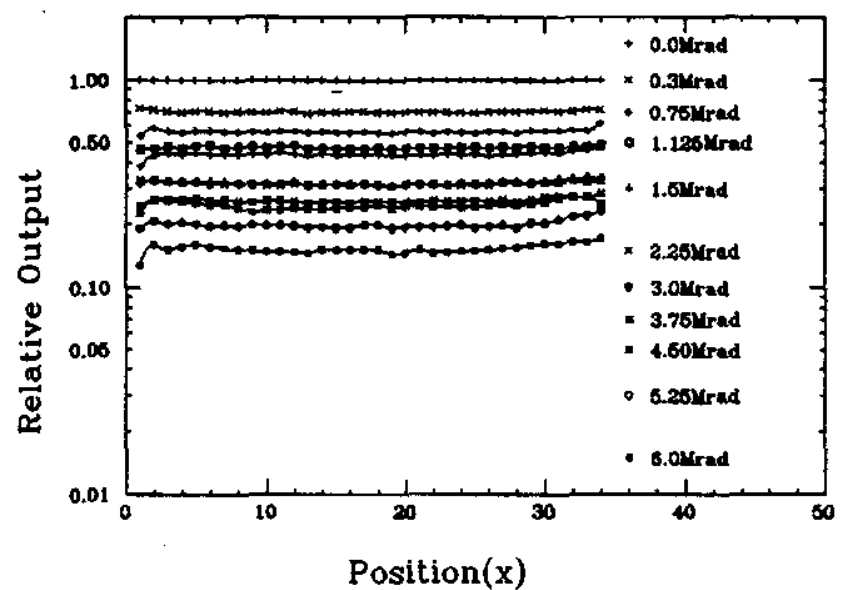

Module \#2 T6 N2

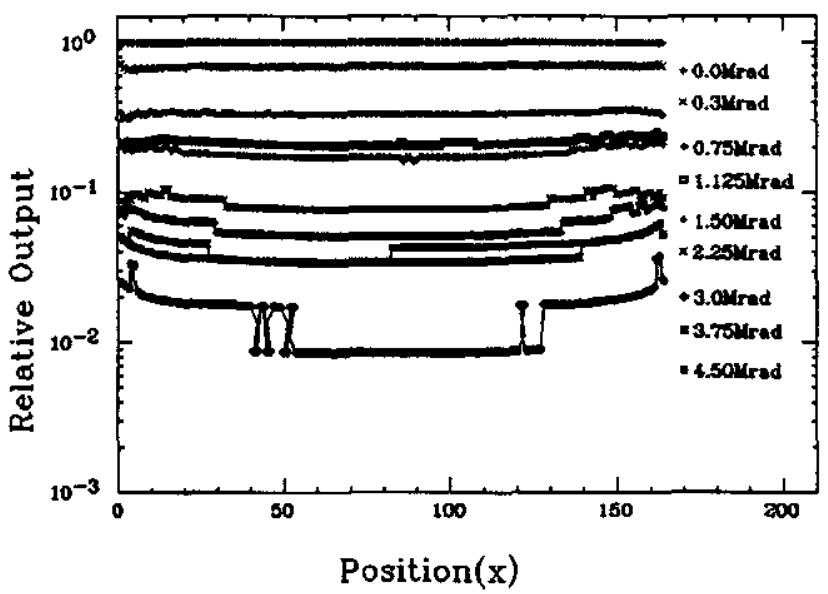

Module \#6 T6 AIR

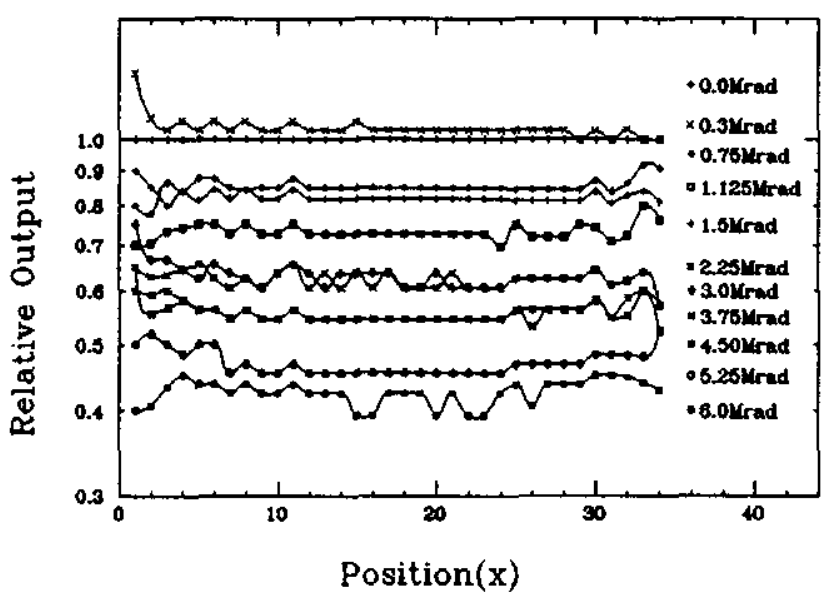

Fig. 6 Relative output (normalized to before-irradiating) at various position $(x)$ across the tile $\# 6$ for module $\# 1,2,5$ and 6 , for different doses. 
Module \#1 T18 N2

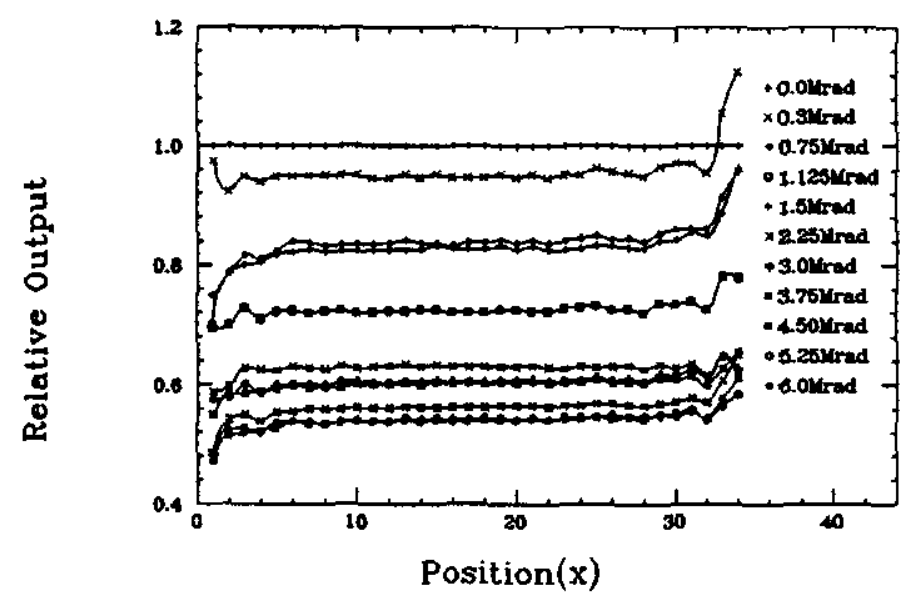

Module \#5 T18 AIR NO BLACK

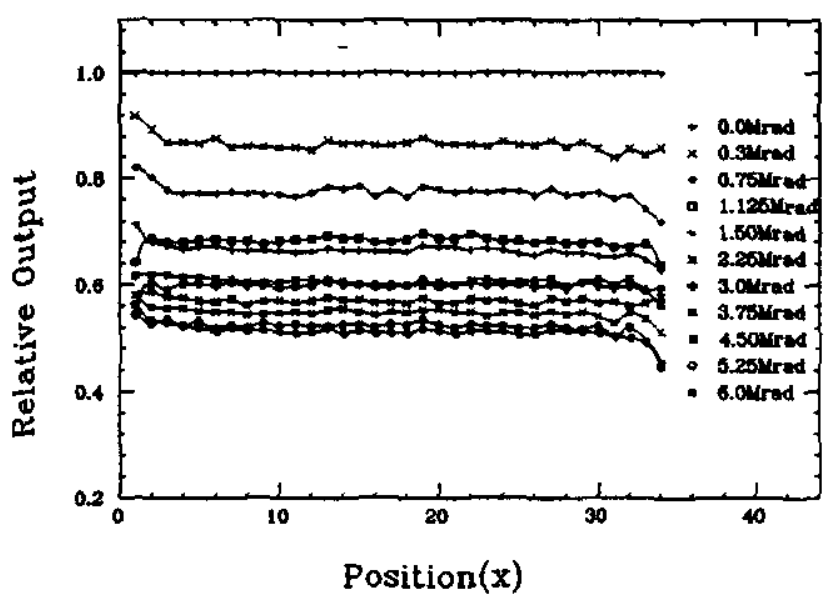

Module \#2 T18 N2
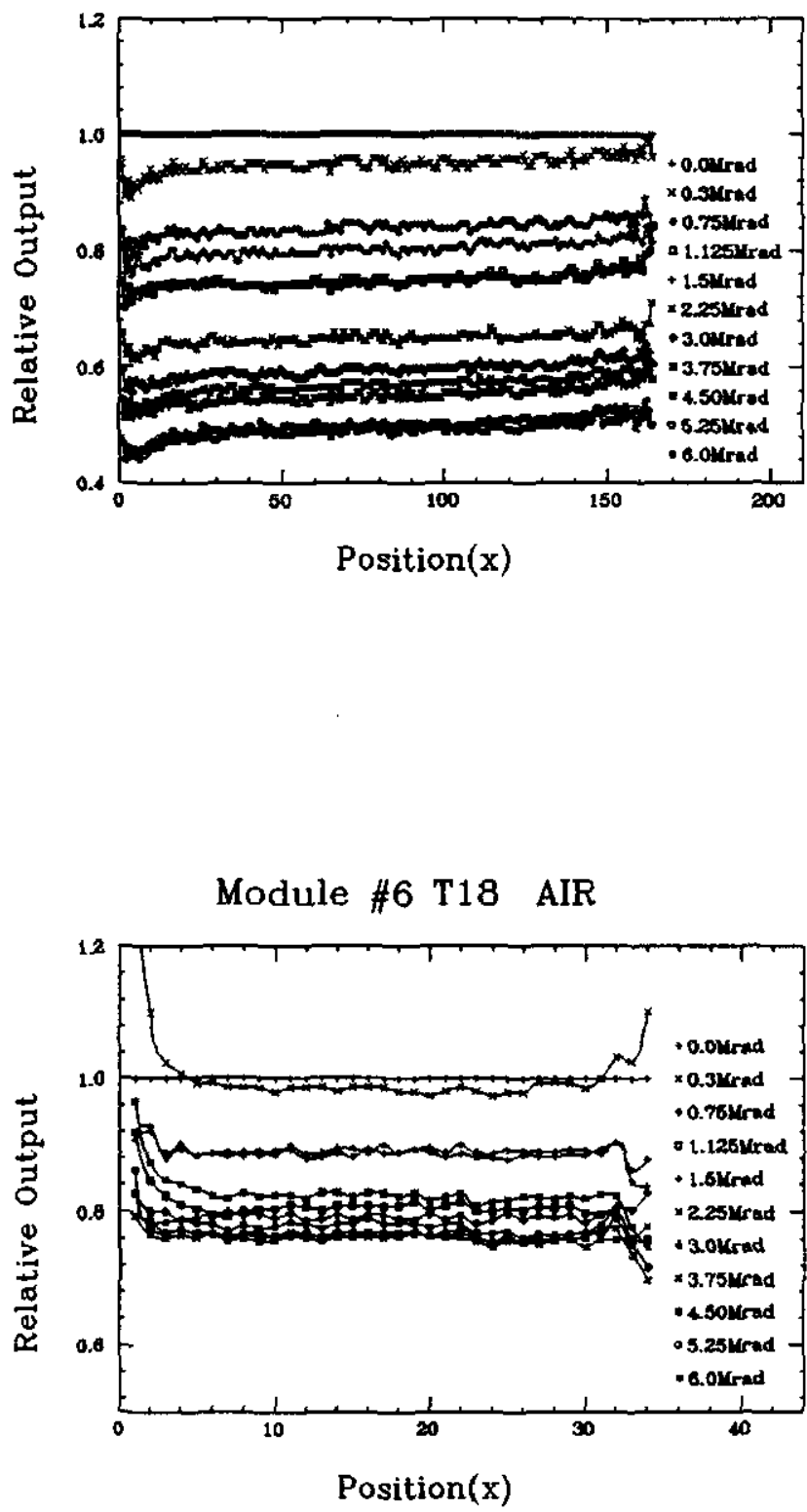

Fig. 7 Relative output (normalized to before-irradiating) at various position ( $x$ ) across the tile 18 for module $11,2,5$ and 6 , for different doses. 


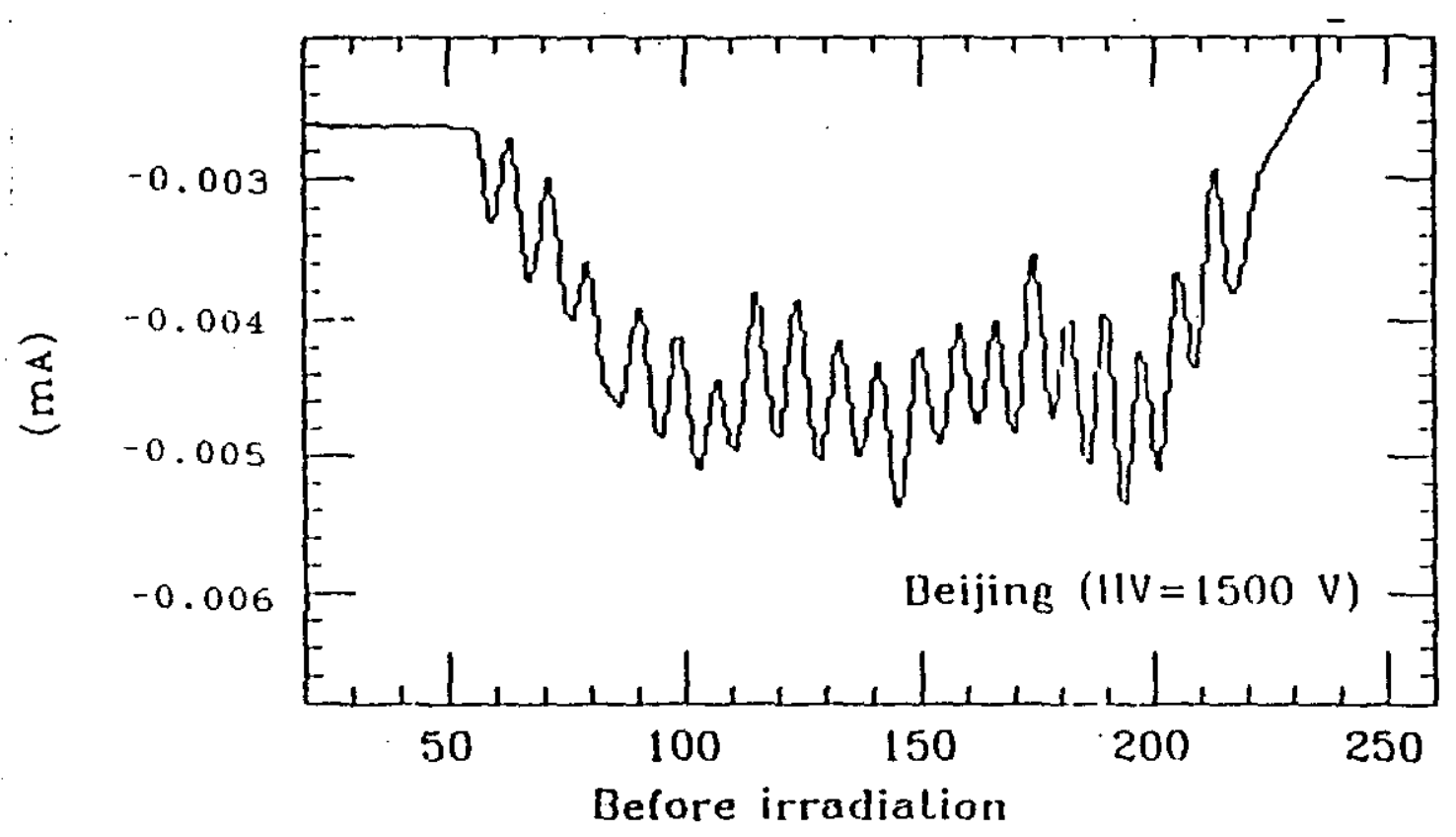

Figure 8. Longitudinal source scan of a test module with 20 layers of scintillator. 
TILE MODULE, \#1 L2 $\mathrm{N}_{2}$

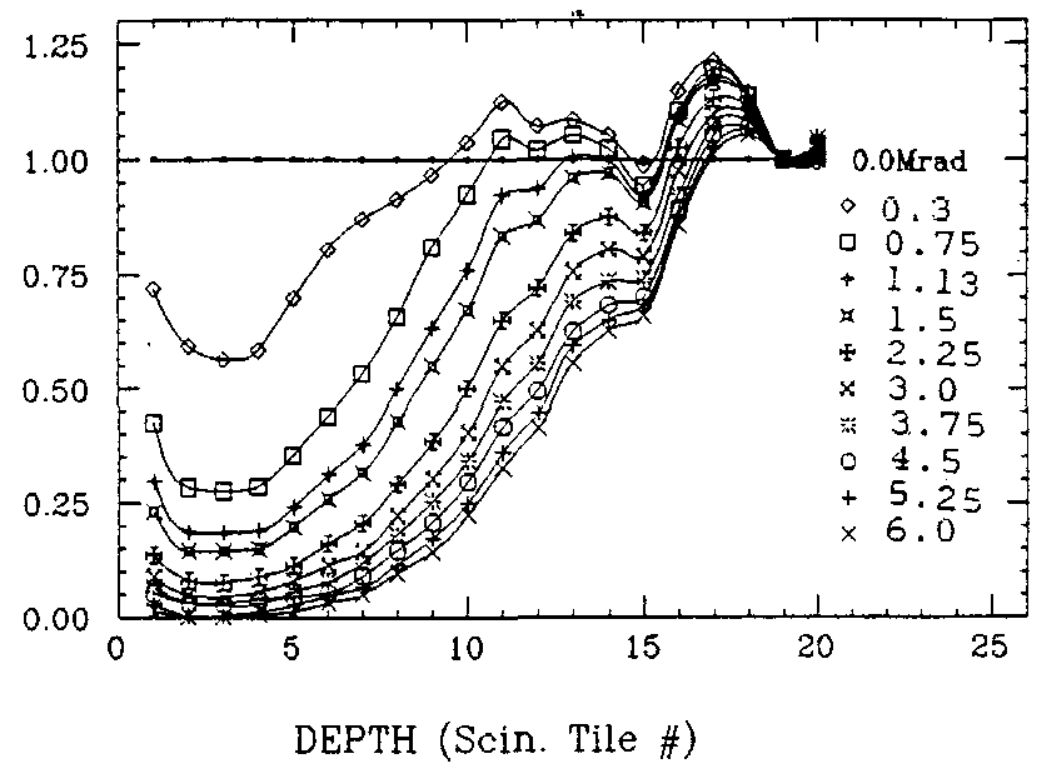

TILE MODULE \#5 L2 AIR

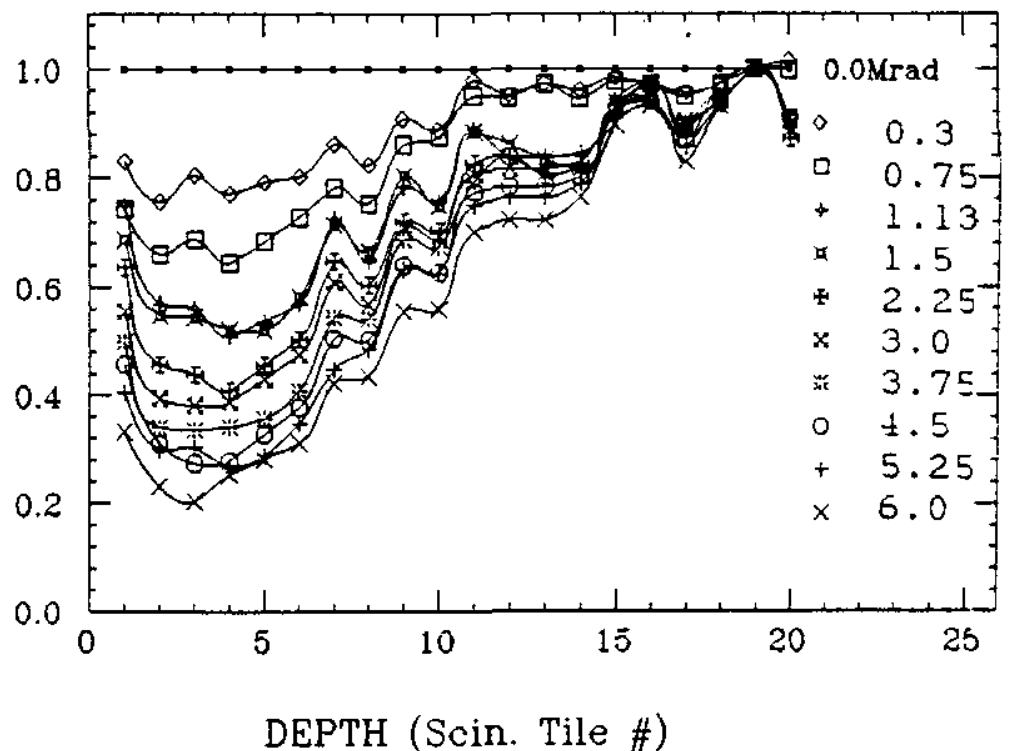

TILE MODULE \#2 L2 $\mathrm{N}_{2}$

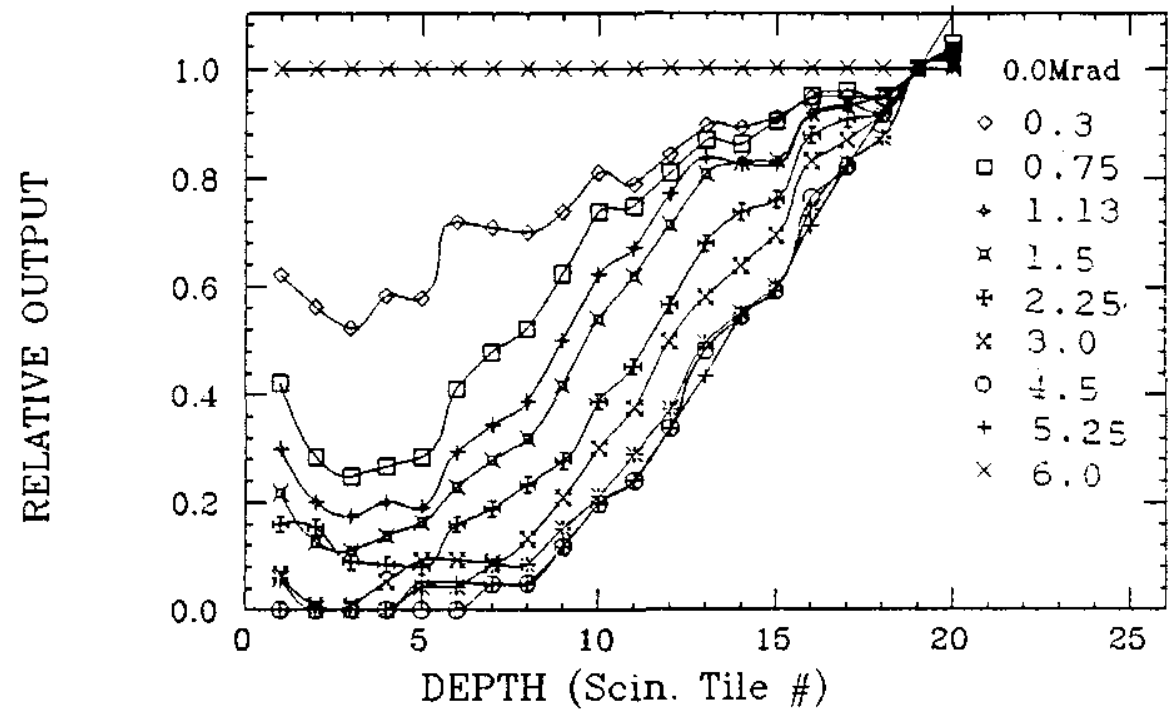

TILE MODULE \#6 L2 AIR

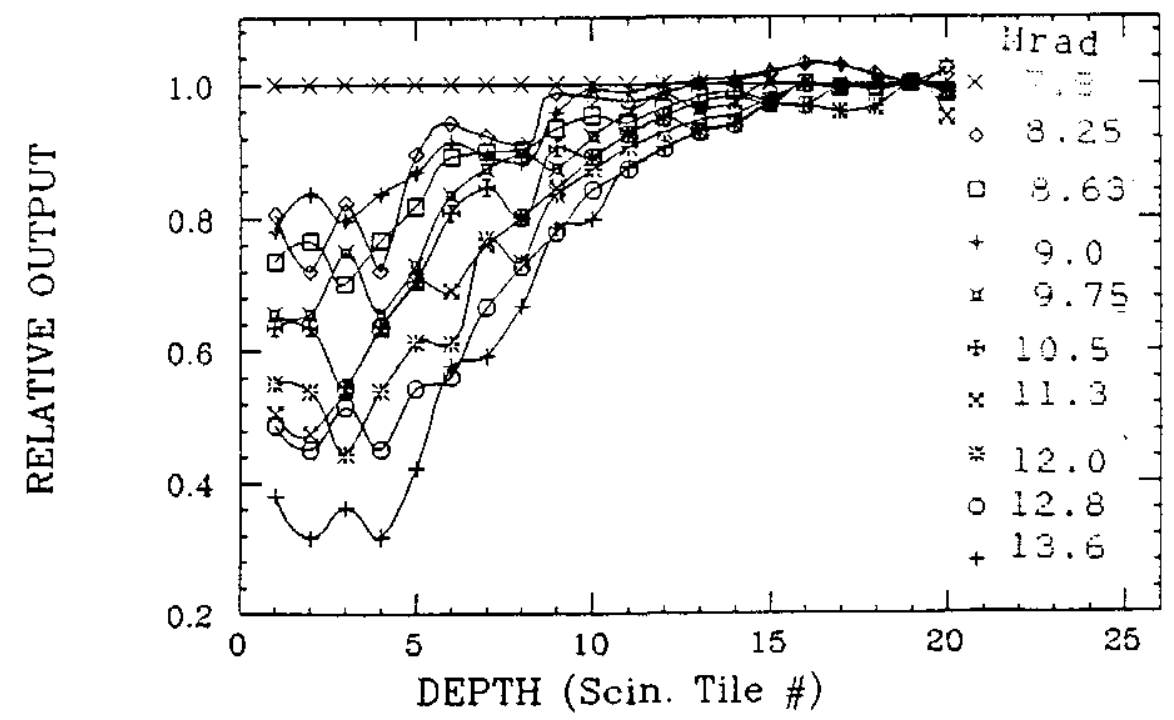

Fig. 9 Depth profile of damage (normalized to peak \#19) for various different doses. 
TILE MODULE \#1 L2 N2,02 RECOVERY

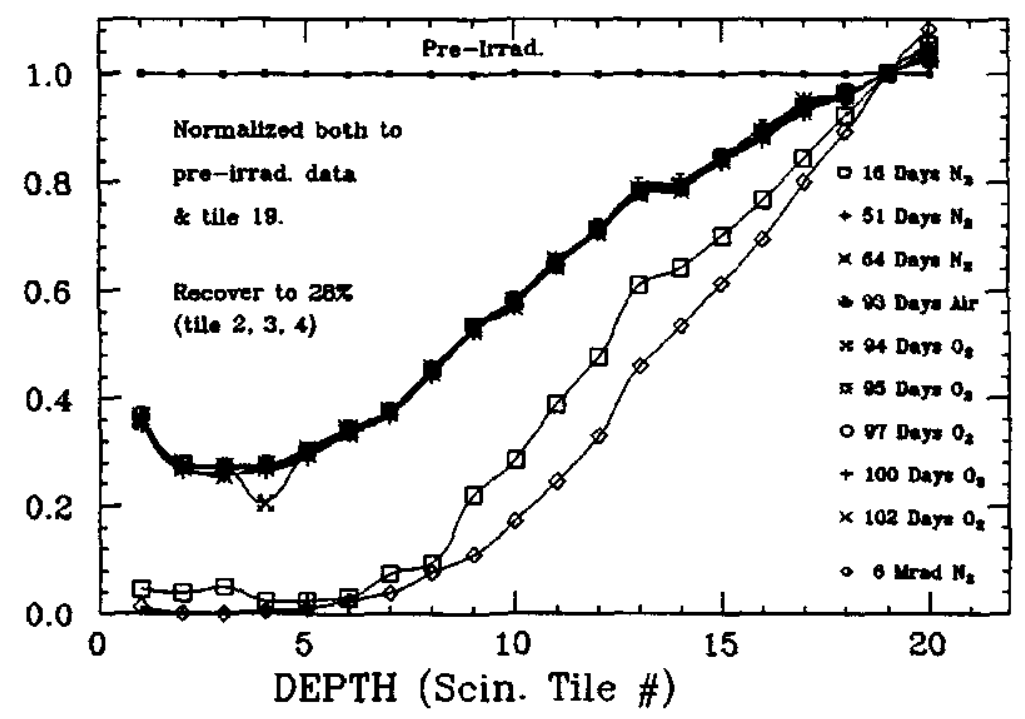

TILE MODULE \#5 L2 AIR RECOVERY

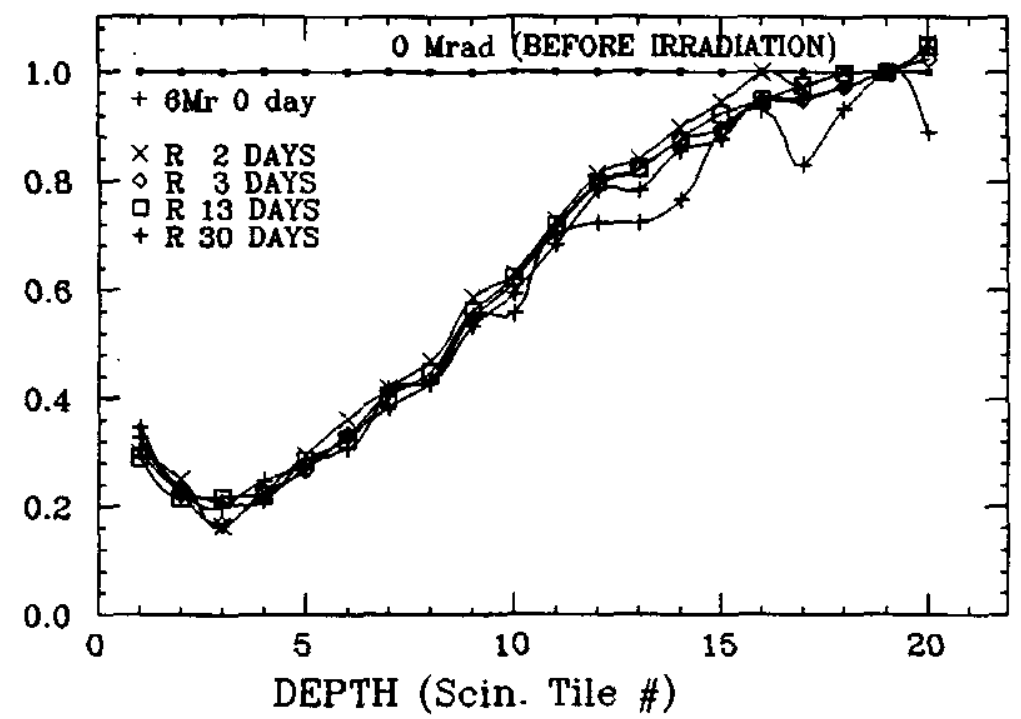

TILE MODULE \#2 L2 N2; AIR RECOVERY

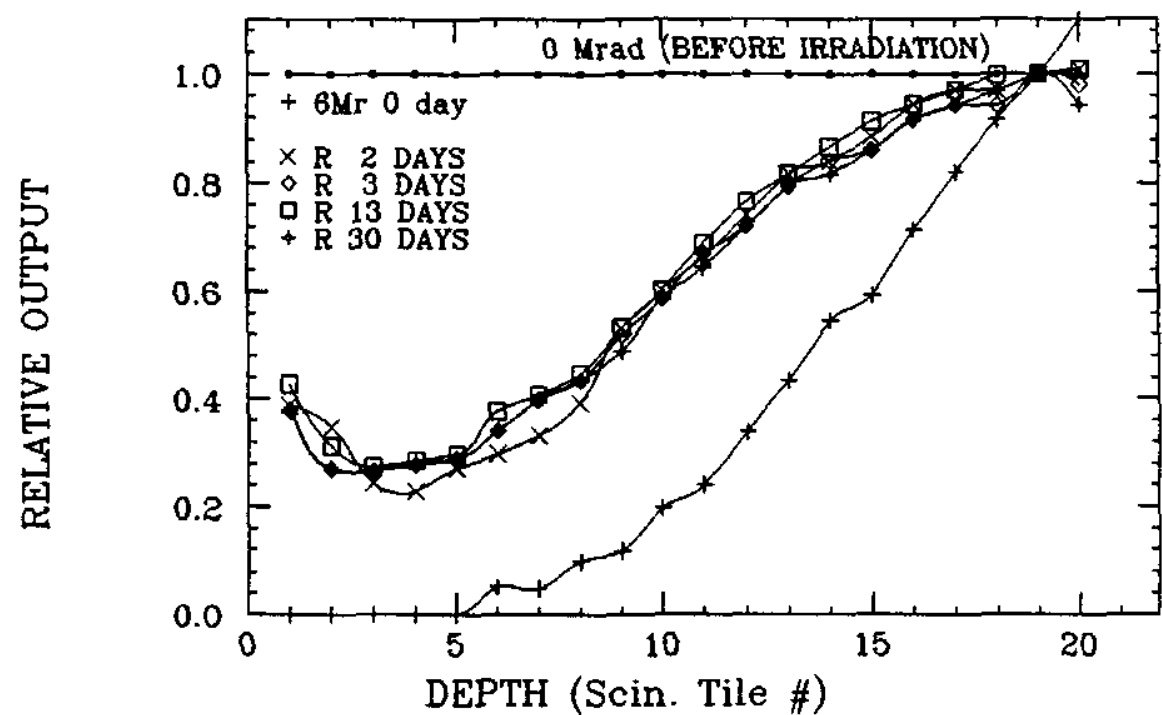

TILE MODULE \#6 L2 AIR RECOVERY

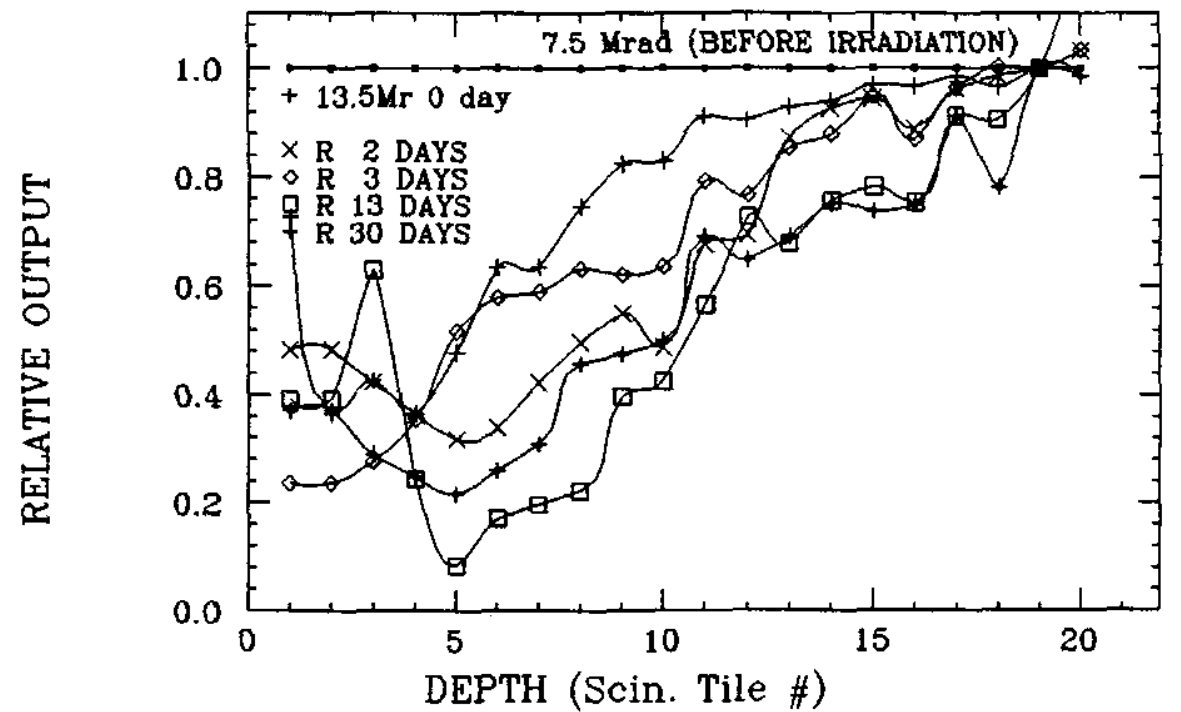

Fig. 10 Annealing plots from longitudinal scan data (Normalized to 0 Mrad \& 'peak' \#19 both) 


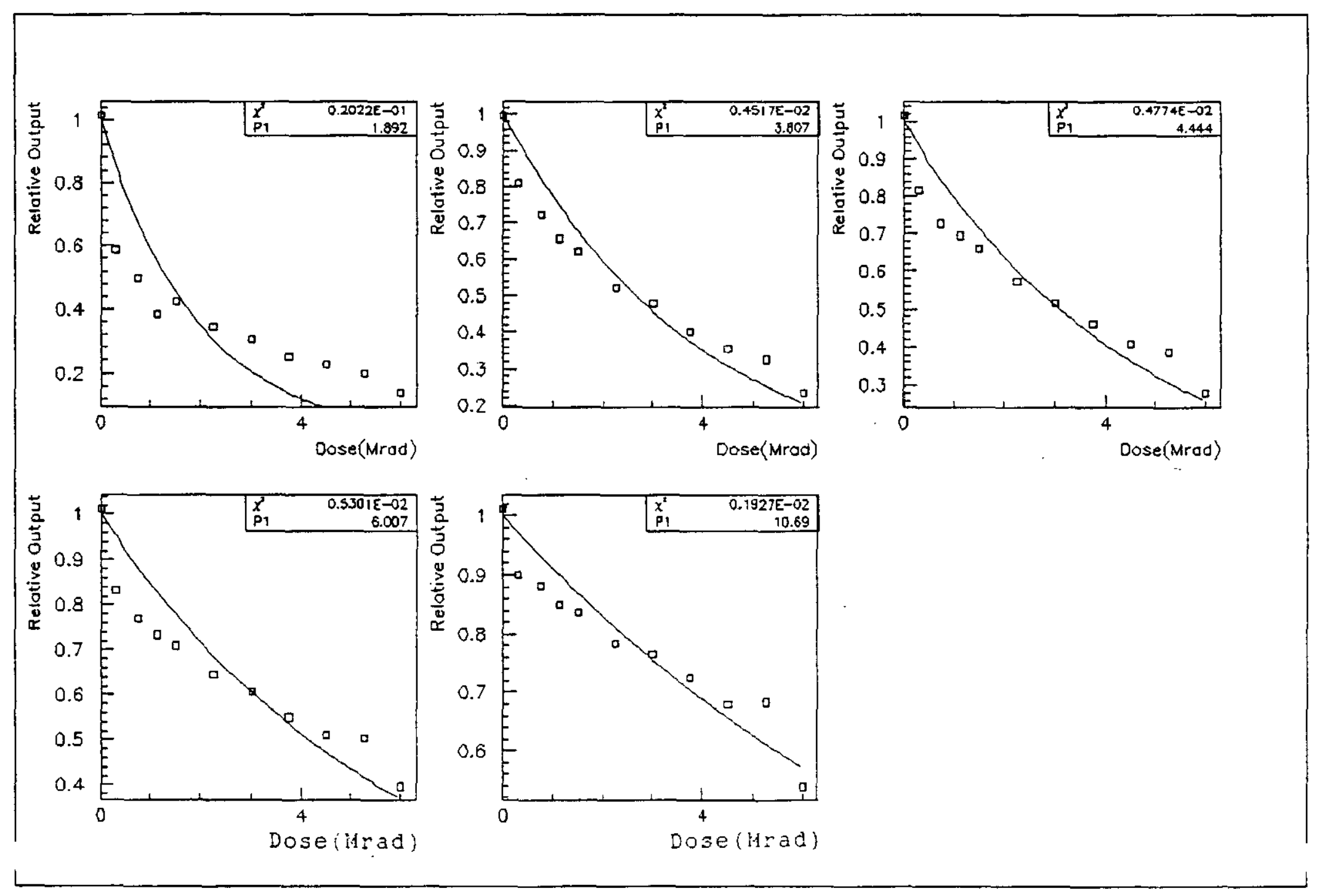




$$
\begin{gathered}
\text { Module \#5 L2 } 1.13 \mathrm{Mrad} \\
\mathrm{F}(\mathrm{x})=\exp (-1 / \mathrm{P} 3((\mathrm{P} 1 * \mathrm{x}) * *(\mathrm{P} 2-1)) * \exp (-\mathrm{P} 1 * \mathrm{x}))
\end{gathered}
$$

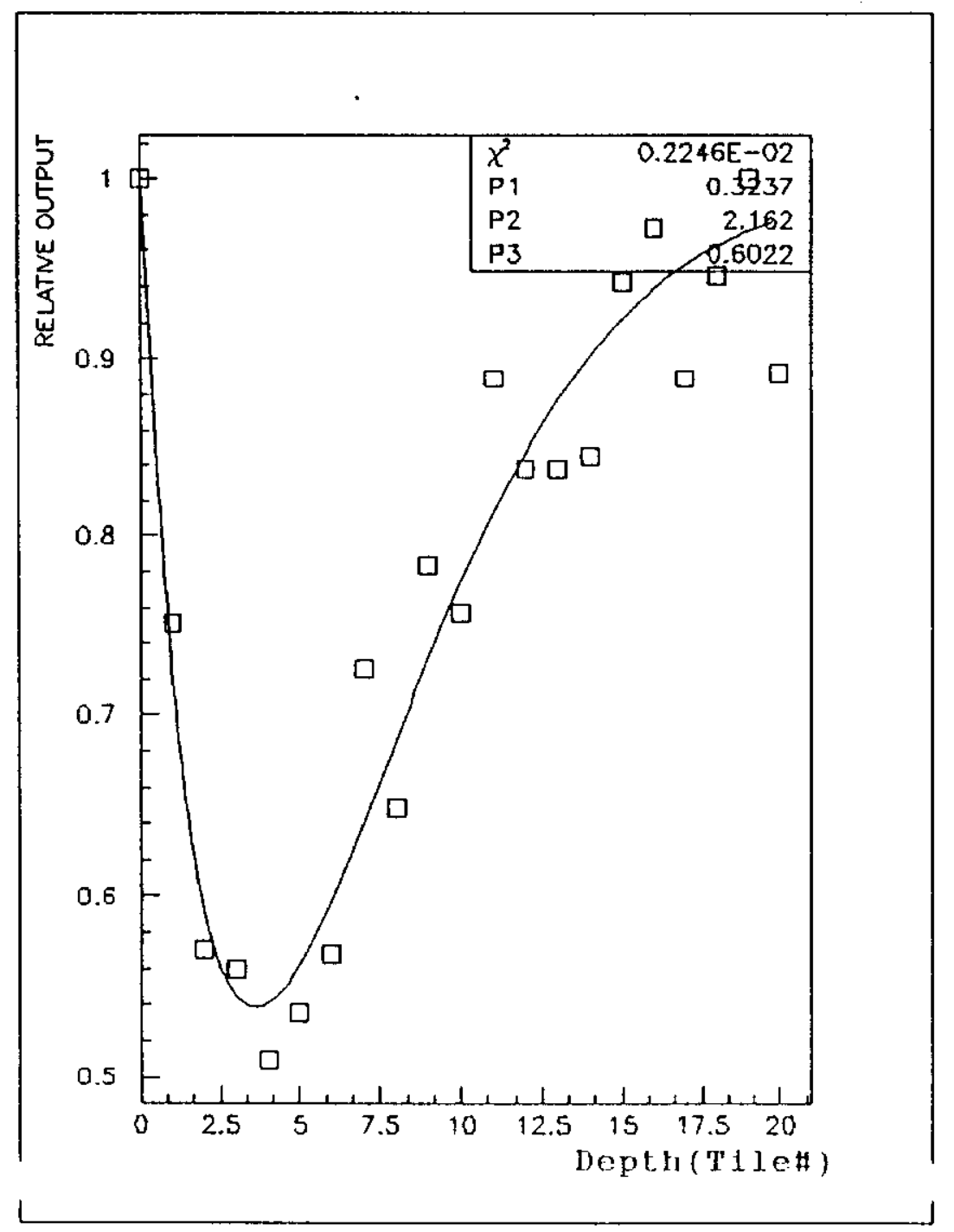

Fig.12 Fitting depth profile curve at $1.13 \mathrm{Mrad}$ 